\title{
N94-11629
}

\author{
Appendix E
}

\section{The Long-Term Dynamical Behavior of Short-Period Comets}

\author{
Harold F. Levison \\ Space Sciences Department \\ Southwest Research Institute, San Antonio, TX 78228 \\ and \\ Martin J. Duncan \\ Department of Physics, Queen's University \\ Kingston, Ontario, Canada K $7 L$ \$N6
}




\begin{abstract}
We numerically integrate the orbits of the known short-period comets mider the influence of Sun and all the planets except Mercury and Pluto. The alculation was undertaken in order to determine the dynamical lifetimes for these objects as well as explaining the current orbital element distribution. It is found that a comet can move between Jupiter-family and Halley-family comets several times in its dynamical lifetime. The median lifetime of the known short-period comets from the time they are first injected into a short-period (omet orbit to ultimate ejection is approximately 50000 years. The very Hat inclination distribution of Jupiter-family comets is observed to become more distended as it ages. The only possible explanation for the observed flat clistribution is that the comets become extinct before their inclination distribution ran change significantly. We show that the anomalous concentration of the argument of perihelion of Jupiter-family comets near 0 and $180^{\circ}$ is a direct result of their aphelion distance being close to 5.2. $4 U$ and the comet boing recently perturbed onto a Jupiter-family orbit. Also the concentration of their aphelion near Jupiter's orbit is a results of the conservation of the Tisserand invariant cluring the capture process.
\end{abstract}




\section{Introduction}

Lnderstanding the origin and evolution of comets is critical to our inderstanding of the origin of the solar system because they are the remnants of the planetesimals that formed Uranus and Neptune and perhaps Jupiter and Saturn. Comets may have also been an important source of the volatiles on the Earth. Short-period comets (those with periods less than 200 years. hereafter SPCs) have been of particular interest recently because of a controversy in the literature concerning their origin. It has been widely believed that SPC. originated in the Oort cloud and evolved into SPC orbits through gravitational interactions with the planets (Newton 1893, see also Everhart 1972). However. in recent years several lines of argument have been put forward that call this iclea into question by showing that it is not possible to reproduce the very flat inclination distribution of the majority of SPCs (Jupiter-family comets with $p<20$ years) from the spherical Oort cloud.

Fernández (10S0) was the first to suggest that these comets originate in lisk of material that lies just beyond the orbit of Neptune. Duncan. Quinn. \& Tremaine (1988, see also Quinu. Tremaine, \& Duncan 1991) have slown that some objects that are initially on low inclination orbits with semi-1najor axes near $50 . A U$ evolve onto orbits that are consistent with the Jupiter-family comets. Thus they argue that this disk of comets is the source for the Jupiter family: Stagg \& Bailey (1989) have presented counter arguments to this idea. In Levison (1991), we argue that this controversy will not be solved until a better understanding of both the current state and evolution of the complete population short-periorl 
becomes available.

It is surprising how little is understood about this population. The sample of short-period comets is effected by observational biases that tend to select objects with small semi-major axes and perihelion distances and perhaps low inclinations (Shoemaker \& Wolfe 1982). Several attempts have been made to correct for these biases (e.g. Shoemaker \& Wolfe 1982, Fernández, Rickman \& Kamel 1992), but the distribution of the complete population is still poorly understoorl.

The long-term behavior of short-period comets are also poorly understood and there are only rough estimates of the dynamical lifetimes of short-period comets. There have been many efforts to study the dynamical behavior of the short-period comets by numerical integration of their orbits (Kazimirchak-Polonskaya 1967, Belyaev (1967), Carusi et.al. 1985. Nakamura d Yoshikawa 1091, and Tancredi \& Rickman 1992). However, these integrations have been limited to times that are much less then the relevant dynamical times, the longest being about 4000 ycars. Rickman (1991) puts the lifetime from first injection into a short-period comet orbit to ultimate ejection at $10^{6}$ years, but does not explain how he arrived at that value.

It is only with the very recent developments in numerical techniques (Wisdom \& Holman 1991) and advances in computer hardware that very long numerical integrations of comets can be achieved. In this paper we unclertale an integration of the orbits of all known short-period comets for as long as 225000 years per comet. We integrate four orbits per comet for a total of 680 orbits. In section 2 we present the numerical techniques. The results of this integration are given in Section 3. Our concluding remarks are in section 4. 


\section{The Integration Techniques}

In this section we discuss the techniques used in our numerical integration of the orbits of all short-period comets (hereafter SPCs) in the Marsden (10S9) Catalog. Their orbits are integrated under the gravitational influence of the Sun and all the planets excluding Mercury and Pluto. The orbits of the Sun and planets are integrated in three dimensions as a full $\mathrm{N}$-body system, in a barycentric frame. The comets themselves are not gravitationally interacting with pach other. We did not include any non-gravitational forces because it is not clear how they vary over such long times periods.

The initial position of the planets were taken from the JPL ephemeris DE200. The initial orbital elements for the $170 \mathrm{SPCs}$ were taken from the Marsden (1989) Catalog. In order to obtain better statistics concerning the behavior of these objects, four orbits were integrated for each comet. One had the initial position and velocity of the real comet calculated from its orbital elements. The other three have initial offsets in position along the $x, y$, or $z$ direction of $0.01 A U$. Since their orbits are chaotic, the four orbits will separate quickly and will soon be independent of one another. In all. the orbits of 680 comets were calculated.

We continue to follow a comet until it either becomes unbound from the Sun and reaches a distance of $50 \mathrm{AU}$. or reaches a semi-major axis greater then $500 \mathrm{AU}$. We integrate the system, including the Sun, planets, and comets, forward in time for 64000 years and backward for 161000 years. At the end of the forward integration two objects remained, while only one remained at the end of 
the backward integration.

We integrate the system using a version of a second order symplectic scheme developed by Wisdom \& Holman (1991) which we modified to handle close approaches. The Wisdom \& Holman method is very fast. It separates the orbit of a body into two parts; the keplerian motion about the Sun and the perturbations due to the planets. Over the timesteps in which a close approach is not taking place, the keplerian motion is known exactly and the perturbations due to the planets are relatively small. Only the perturbations need to be numerically integrated. Thus the timestep used can be much larger than that for previous methods which must also numerically integrate the keplerian orbit about the Sun. For details on our corle see Duncan. Levison. \& Zingle (1993).

We have found that to reach a adequate accuracy using the symplectic integrator of Gladman \& Duncan (1990) requires hundreds of timesteps per orbit, while the Wisdom \& Holman technique described by Duncan, Levison, S Zingle (1903) only requires tens of timesteps. In practice we found the new technique is approximately an order of magnitude faster than traditional methods.

We have modified the Wisdom \& Holman technique to accurately integrate close approaches between a comet and a planet. To do so we define two zones about each planet. The inner zone is defined to lie within one Hill radius, conventionality defined as (c.f. Lissauer 1993)

$$
r_{h}=a_{p}\left(\frac{1}{3} \frac{M_{p}}{M_{0}+M_{p}}\right)^{\frac{1}{3}},
$$

of the planet, where $a_{p}$ is the semi-major axis of the planet in $A U$, and $M_{\odot}$ and $M_{p}$ is the mass of the Sun and planet respectively. The outer zone is defined to 
lie between one and three Hill radii. If a comet lies within the outer zone at the beginning of a timestep or is predicted to lie within this zone at the end of the timestep then its timestep is clecreased by a factor of 100 . If a comet lies within the inner zone at the beginning of a timestep or is predicted to lie within it at the end of the timestep then its timestep is again decreased by a factor of five. Also in the inner region, when the orbit of the comet is separated into the two parts, the keplerian part is centered about the planet rather than the Sun.

The unmodified technique is well tested by Wisdom \& Holman (1902). We test on modification that handles close approaches by integrating the orbits of several massless particles in the planar restricted three body problem. The massive particles have the mass of the Sun and Jupiter and are in a circular orbit about each other with a semi-major axis of 5.2.AU. The test particles are all on Jupiter crossing orbits with semi-major axes between 4 and $6 . A U$. The integration lasts for 6000 years. On average, a particle suffers 45 close approaches with Jupiter and its Jacobi integral is conserved to better than one percent during the cutire integration. In the most extreme encounter seen, the particle passes within $6 \times 10^{-4} A U$ or 0.7 Jupiter radii from the planet. During this encounter its Jacoli constant is conserved to one part in $4 \times 10^{-6}$. Thus, we think the code performs adequate for our purpose especially since the comets are all on planet crossing orbits and are very chaotic. 


\section{Results}

Before presenting the results of the integrations we must first caution the reader. Since the orbits are planet crossing and thus chaotic, it is not possible to accurately determine the long-term fate of any individual comet. However, it is appropriate to extract statistical information from these integrations about the behavior of this sample of comets that will resemble the evolution of the real system. For the remainder of this paper we only discuss the statistical attributes of our integration.

Using our integration, it is possible for the first time to directly calculate the dynamical lifetimes of SPCs. Figure 1 shows the fraction of comets with lifetimes greater then a particular time as a function of that time. We plot the total lifetime combining both the forward and backward integrations. The median lifetime is 48000 years. Three of our comets have lifetimes greater than $2 \times 10^{\text {is }}$ years. Figure 1 shows that the population follows an exponential decline until approximately $80 \%$ of the comets are removed. The exponential decay time for the system in approximately 74000 ycars. The remaining $20 \%$ of the comets are longer lived than a simple exponential decay would predict.

It has been typical in the literature to divide SPCs into two families; Jupiter and Halley family comets (for example see Carusi \& Valsecchi 19S7). Here we define a Jupiter family comet (hereafter JFC) as one with a period less than 20 years and a Halley family comet ( $\mathrm{HFC}$ ) as one with a period between 20 and 200 years. The two families can be distinctly seen in Figure 2, which shows the inclination of the comets in the Marsden catalog as a function of their 
semi-major axis. The dotted line represents the division between JFCs and HFCs. It can be clearly seen in the figure that the JFCs have a much flatter inclination distribution than the HFCs. The median $\cos (i)$ of the JFCs is 0.985 (corresponding to $i=10^{\circ}$ ) while the median $\cos (i)=0.62\left(i=52^{\circ}\right)$ for HFCs.

JFCs have two interesting characteristics in their orbital element clistribution that still must be understood. These are shown as histograms in Figure 3. Figure 3a shows a histogram of the aphelion distances of JFCs, $Q$, which are strongly peaked near the semi-major axes of Jupiter. Approximately $73 \%$ of them have $Q$ between 4.2 and $6.2 A U$. Figure $3 b$ shows a histogram of the their argument of perihelion. $w$. It can be seen that the argument of perihelion is strongly concentrated near 0 and $180^{\circ}$. Approximately $75 \%$ of linown JFCs have $\omega$ within $45^{\circ}$ of these two values. A value of $\omega=0$ or $180^{\circ}$ implies that when the comet is in the plane of the solar system, it is also at either perihelion or aphelion. We propose an explanation for these observations later in this section.

It has been argued that much of the inclination difference may be duc to (b)ervational biases in the discovery of these objects. Shoemaker (1092) has most fully cleveloped these arguments. As we will see, he concludes that they cannot be entirely responsible for the observed inclination distribution of JFCs. Since his work is unpublished we reproduce lis argument here. Shoemaker points out that most JFCs were discovered during searches for asteroids. These surveys were primarily performed only near the ecliptic. On the other hand, most HFCs were liscovered by amateurs who survey near the Sun at all inclinations. Although this would naturally explain the observed inclination differences. he argues that observational biases will be much less important for comets with small perihelion 
distances. q. Thus in Figure 2 we distinguish comets with $q \leq 1 A U$ (plotted as filled circles) from those with larger values of $q$ (open circles) in order to remove these biases. As the figure illustrates, the inclination distributions are still very different for this subset of objects. The median $\cos (i)$ for this subset is $0.98(i=$ $\left.11^{\circ}\right)$ for $\mathrm{JFCs}$ and $0.12\left(i=83^{\circ}\right)$ for HFCs. Therefore, the difference seen for the complete sample cannot be entirely due to observational biases (Shoemaker 1992).

The very flat inclination distribution of the JFCs has been used to argue that JFCs and HFCs are dynamically distinct systems that have different origins. Quinn. Tremaine. \& Duncan (1991) argue that while HFCs are most likely captured long-period comets, it is not possible to reproduce the very flat distribution of JFCs in this manner. They argue that JFCs must have come from a source that is intrinsically flat. They suggest that there is a belt of comets beyond the orbit of Neptune that is the source of JFCs, the Kuiper belt.

However, our integration slows that JFCs and HFCs are not dynamically distinct objects. Indeed, a comet can move between the families several times in its history. Figure 4 shows the dynamical evolution of the comet P/Parker-Hartley. We present this figure only to illustrate the possible behavior of a comet. Because the orbit is chaotic, this figure cannot be used to predict the long-term behavior of this particular comet. In our integration $\mathrm{P} / \mathrm{Parker}-\mathrm{Hartley}$ has a dynamical lifetime of 83000 years. Figure 4 a shows the evolution of its semi-major axis over its entire lifetime. The dashed line represent the boundary between JFCs and HFCs. Notice the the comet moves back and forth across this boundary several times. A SPC in our integration crosses this boundary on average 8.3 times and at most 58 times in its dynamical lifetime. 
Figure $4 \mathrm{~b}$ shows the evolution of the perihelion distance of comet P/Parker-Hartley. Quinn, Tremaine, \& Duncan (1991) define a 'visible' comet ns one with $q \leq 2.5 \mathrm{AU}$. If a comet has a $q$ greater than this value then, they argue, it will not become bright enough to be cliscovered. The dashed line in the figure represents this visibility boundary. Notice that the comet becomes visible and invisible several times during its lifetime.

Figure $4 \mathrm{c}$ shows the behavior of the inclination of comet over its lifetime. Its inclination varies significantly. Indeed, at $\$ 220$ years in the future the orbit becomes retrograde. It remains retrograde for 1000 years before it is ejected. During that time it is a visible JFC! Interestingly, there are no known visible JFCs on retrograde orbits. This is somewhat surprising because our integration shows that the typical SPC in our sample spends approximately $2.2 \%$ of its time as a visible retrograde JFC. Thus, it predicts that there should be approximately four such objects in the real sample of comets. (Note that low inclination retrograde comets would not be affecter by the observational biases discussed ubove. We return to this problem later in this section.)

Figure 4d shows the temporal behavior of the argument of perihelion of comet $\mathrm{P} /$ Parker-Hartley. There is no tendency for the comet to have $\omega$ close to 0 or $180^{\circ}$. Indeed, between approximately 30000 and 70000 years in the past the comet appears to circulate in $\omega$.

In our initial sample of comets, $87 \%$ are JFCs. We find that $90 \%$ of all our comets become JFCs at some point in their lives, thus 5 of the known comets that are currently HFCs will become JFCs in their lifetimes. The average comet in our integration spends $53 \%$ of its time as a JFC and $82 \%$ of its time with 
$i<40^{\circ}$. It spends $42 \%$ of its time visible; $28 \%$ of its time as a visible JFC and $10 \%$ of its time as a visible HFC. By 'visible' we mean that the comet has $q<2.5 \mathrm{AU}$, we do not distinguish between active and extinct comets in this context.

\section{a) Inclination Distribution of JFCs}

In this subsection we discuss the origin of the very flat distribution of observed Jupiter-family comets, as illustrated in Figure 2. Figure 5a shows the mean cosine of the inclination of all comets remaining as a function of time for both our forward and backward integrations. Figure jb shows this value for just the JFCs. The solid line shows all JFCs while the dotted line shows only the visible JFCs. The system clearly becomes much less flat as it evolves.

Notice that the inclination distribution fattens up in our backward integration as well as our forward one. This is due to the chaotic nature of the orbits. In an infinitely accurate calculation with infinitely precise initial conditions, all our comets could be traced back to their origin. Since the orbits are chaotic and diverge exponentially in time, it is not possible to recover their initial distribution. Also since it is statistically more likely for objects to evolve to higher inclinations, we observe this increase in both our forward and backward integrations.

What is the cause of the flat inclination distribution for JFCs? As stated above, Quinn, Tremaine, \& Duncan (1991) argue the high inclination JFCs do not exist. Indeed, their argument in support of the liuiper belt being the source of JFCs is a result of the fact that these comets are on low inclination 
orbits. However. Shoenaker (1992) points out that meteor streams are observed (Olsson-Steel 1988) that have semi-major axes similar to JFCs but have high inclinations. Thus, he argues that high inclination JFCs must exist, but have remained undiscovered. Understanding the origin of these high inclination objects is pivotal to whether the Kuiper belt is the source of JFCs.

Shoemaker argues that the low inclination visible JFCs and the hypothetical invisible high inclination. JFCs are part of the same population and that they may evolve from one into the other. His argument is based on the Tisserand invariant which is defined as

$$
T=\frac{a_{J}}{a}+2 \sqrt{\frac{q}{a_{J}}(1+\epsilon)} \cos i,
$$

where $e$ and $q$ are the eccentricity and perihelion distance of the comet, and, $a$ and $a_{J}$ are the semi-major axes of the comet and Jupiter respectively. If $T$ is approximately conserved for JFCs and their semi-major axes rlo not vary significantly, then there is a relationship between a comet's perihelion distance and its inclination. Objects with low inclinations will have smaller values of $q$ than those with high $i$. Thus, since the actirity of a comet is a function of its perihelion distance, low inclination objects will be more active. Shoemaker argues that JFCs are not only on low inclination orbits. but that only.JFCs with low inclinations come close enough to the Sun to become visible.

If this theory is correct then the current observed sample of JFCs are biased toward low inclinations. This predicts that if we integrate the orbits of the comets, the population will phase mix and the inclination distribution of the comets will come to match that of the real distribution of unbiased JFCs, i.e. the system will fatten up. This is what we observe in the integration (Figure 5). 
However, there is a second prediction of the theory. Since the semi-major axes and the Tisserand invariant are approximately the same for all JFCs, then this theory predicts that there should be a correlation between a comets inclination and its perihelion distance. In particular, objects with large $q$ should have high $i$. We test this hypothesis by first dividing perihelion distance into 10 equally spaced bins for $q<5 A U$. During the integration we record the inclination of every comet as a function of which bin it is in. Figure 6a shows the mean cosine of the inclination of all comets within a bin as a function of the bin's mean perihelion distance. The filled circles represent the mean for all comets within the bin, while the open circles represent the mean for only those comets the are on direct orbits. If this theory were correct we would find that larger values of $q$ would imply larger $i$. This trend is not observed. Thus, Shoemaker's conjecture is not consistent with our integration.

There are two other possible explanations for the increase in inclination observed in our integration. Firstly, low inclination objects may have shorter rynamical lifetimes than objects with large $i$. They would be removed first from the system and thus its mean inclination would increase. To test this possibility, we plot the mean inclination of a individual comet as a function of its lifetime in Figure 6b. If this scenario were true, we would expect to see a correlation between a comet's lifetime and its inclination. which is not observed.

The final explanation is that the orbits of individual comets may tend to evolve to higher inclinations. In this scenario, the decrease in the mean $\cos i$ observed in the system is a result of dynamical relaxation. If this were correct then we would expect that on average the mean inclination that a comet has 
cluring its lifetime would be larger that its current inclination. In Figure $6 \mathrm{c}$ we plot these two values for the 680 comets in our integration. Notice that most 'omets fall above the diagonal line, implying that on average the inclination of a comet increase with respect to time. Thus it appears that the reason that the mean inclination of our sample of comets increases is because the inclination of individual comets tend to increase.

So, why is the observed distribution of JFCs so flat? We suggest that it is because comets become extinct on timescales that are much shorter than their dynamical lifetimes. If this is the case then the JFC's that are observed are objects that only recently became active for the first time. Comets must be on low inclination direct orbits when they initially become JFCs. The comets fade and become extinct before their orbital elements can change significantly. This explains the lack of high inclination active comets. It also allows for the existence of the high inclination meteor streams because it predicts that extinct JFCs are on average on higher inclination orbits than active ones.

This idea is consistent with several previous numerical integrations (Tiarm it Rickman 1982, Tancredi \& Lindgren 1992) which show that most JFCs have recently had a close approach with Jupiter. It also agrees with the two main results of Quinn. Tremaine, \& Duncan (1991). Our argument that JFCs must come from comets on low inclination orbits agrees with their notion that the Kuiper belt is the source of these objects. They also concluded that HFCs and JFCs must have different sources. We stated earlier that this seems inconsistent with our integrations because comets easily evolve from one family to another. However, if they become extinct before most of them can evolve much in 
semi-major axis then it is possible for the two families of active comets to have different origins.

\section{b) Other Orbital Elements}

We now return to the origin of the other orbital elements of the known JFCs. Their distribution is strongly concentrated so that $Q \approx 5.2 A U$ and the perihelion is near the ecliptic ( $\omega \approx 0$ or $\left.180^{\circ}\right)$, see Figure 3 . Figures 7 a and $7 b$ show the $Q$ and $\omega$ distribution for visible JFCs at various times in our integration. The solid histogram shows the current distribution. The dotted histogram represents the distribution averaged over 2000 years centered on 5000 ycars in the future and dashed histogram is averaged over 20000 years centered on 50000 years in the future. The amplitude of the peaks in both figures decrease by nearly $50 \%$ in 5000 years, but remain approximately constant after that. This implies that the observed distribution does not represent a steady state distribution but is biased toward the peaks seen in the figures.

As with the inclination distribution, the only possible explanation for the observed strong concentration of orbital elements is that the observed JFCs are objects that have only recently become active for the first time. They must fade and become extinct before their orbital elements can change significantly. The observed distribution must therefore be a result of the mechanism that produces visible JFCs.

We explain the observed distribution in two steps. First we explain the $Q$ distribution. Then we show that the distribution of $\omega$ is a natural result of $Q$ being close to $5.2 \mathrm{AU}$ and the comet being recently perturbed onto a JFC orbit. 
A visible JFC is most likely produced when a comet suffers a close approach with Jupiter. Indeed, Karm \& Rickman (1982) have shown that most JFCs have recently undergone such an experience. During this encounter the Tisserand invariant, $T$, (equation 2 ) is approximately conserved. Thus the value of $T$ of a JFC when it becomes visible must be approximately the same as it was just before the encounter.

A comet coming in from the outer regions of the solar system is most likely to be perturbed onto a JFC orbit if its perihelion distance. $q$, is close to Jupiter's semi-major axis. $a_{j}$. If we assume that before the encomter $q=a$, and $\cos (i)=$ 1. then the Tisserand invariant beromes

$$
T=\frac{1}{a_{i}^{\prime}}+2 \sqrt{2-\frac{1}{a_{i}^{\prime}},}
$$

where $a_{i}^{\prime}=a_{i} / a_{J}$ and $a_{i}$ is the initial semi-major axis of the comet. The assumption that $\cos (i)=1$ is well justified by the fact that the median cosine of the inclination of JFCs is 0.955 . We can solve equation 2 for the erentricity: $r_{f}$, of the orbit after the encounter as a function of the $q_{f}$. We find

$$
e_{f}=1+2 q_{f}^{\prime 3}-q_{f}^{\prime} T+2 q_{f}^{\prime 3 / 2} \sqrt{2-q_{f}^{\prime 3}-q_{f}^{\prime} T}
$$

where $q_{f}^{\prime}=q_{f} / a_{J}$ and again we assume the final $\cos (i)=1$. We can find a relationship between the final aphelion distance. $Q_{f}$, and $q_{f}$ and $a_{i}$ by using the general result

$$
Q=q \frac{1+e}{1-e}
$$

This relationship is shown in Figure 8a, where we plot the final aphelion distance of a comet as a function of its final perihelion clistance for four different 
values of $a_{i}$. Since we are only interested in visible orbits, we only plot $q_{f}<$ 2.5AU. The three smaller values of $a_{i}$ were chosen so that the initial $Q$ was lose to the semi-major axis of one of the 3 other giant planets. The largest $a_{i}$ was chosen to represent objects from the Oort cloud. Since it is not possible for $Q_{f}<a_{J}$, there is a minimum $Q_{f}$ for each value of $a_{i}$. Indeed, the figure shows that this simple argument predicts that it is not possible for a comet with $q$ initially near Jupiter and $Q$ near Saturn to evolve onto an orbit with $q_{f}<2.5 \mathrm{AU}$. Also it predicts that it is not possible for $q_{f}<1 A U$. The largest possible value for $Q_{f}$ is $6.4 A U$, which corresponds to $a_{i}>>a_{J}$ and $q_{f}=2.5 . A L$. Thus, this argument predicts that $5.2 \leq Q \leq 0.4 . A U$. This range is shown by the arrows in Figure 7 a. It agrees very well with the peaks in the observed $Q$ distribution (solid histogram in Figure $7 \mathrm{a}$ ). It is interesting that this argument breaks down if the perihelion distance before the encounter is not close to $a_{J}$. We can reverse the argument and claim that the observed $Q$ distribution implies that $q_{i} \approx a_{J}$.

The observed distribution in $\omega$ (the solid histogram in Figure $て$ ) is a direct result of the $Q$ distribution and the comet's recent dynamical history. Since Jupiter-family comets have recently suffered a close approach to Jupiter. the comet must be close to the ecliptic when it is $5.2 . A U$ from the Sun. Independent of our previous argument, it is an observed fact that JFCs tend to have $Q$ only slightly larger than 5.2AU. This implies that the comet must be close to aphelion when it is in the ecliptic. On the other side of its orbit, it will be close to the ecliptic when it is at perihelion. So, $\omega$ must be near 0 or $180^{\circ}$.

We can quantify this argument. The equation of a keplerian orbit is

$$
r=\frac{a\left(1-e^{2}\right)}{1+e \cos \nu}
$$


where $r$ is the instantaneous distance between the Sun and the comet and $\nu$ is the true anomaly. Since the comet has recently experienced a close approach with Jupiter, it must be on an orbit that intersects the orbit of Jupiter. This implies that the comet must be on the ecliptic when $r=a_{J}$. By definition, this occurs when the comet is at one of the nodes. If it is at the ascending node then $\nu=-\omega$, if it is at the descending node then $\nu=180^{\circ}+\omega$. In equation 5 we can replace $r$ with $a_{J}$ if we replace $\cos \nu$ with $\cos \left(\omega+\omega_{o f f}\right)$, where $\omega_{o f f}$ is either 0 or $180^{\circ}$. So

$$
\cos \left(\omega+\omega_{n f f}\right)=\frac{1}{\epsilon}\left[\frac{a}{a_{j}}\left(1-c^{2}\right)-1\right] .
$$

We can now apply this relationship to the orbit of our hypothetical comet after its encounter with Jupiter and produce a relationship between $\left|\omega+\omega_{0 f f}\right|$ after the encounter to $a_{i}$ and $q_{f}$. This relationship is shown in Figure sb. The largest possible value for $\left|\omega+\omega_{n f f}\right|$ is $46^{\circ}$, which corresponds to $a_{i} \approx \infty$ and $q_{f}=2.5 .4 U$. This implies that $\omega$ must be within $46^{\circ}$ of 0 or $180^{\circ}$. These limits are shown by the arrows in Figure $7 b$. They agrees very well with the peaks in the observerl $w$ distribution (solid histogram in Figure $7 \mathrm{~h}$ ). Note that the distribution would be more concentrated if $a_{i}$ were within the planetary region, say less than $50 . A U$.

In this subsection we showed that the concentration of the argument of perihelion of JFCs toward 0 and $180^{\circ}$ is a direct result of their $Q$ being near $5.2 A U$ and the fact that their orbits intersect the orbit of . Tupiter. These conditions are observed to be true for the known. JFC population. Thus, the validity of our argument is independent of whether we understand the mechanism that produces these conditions. We have also shown that these conditions arise if a comet was recently captured onto a JFC orbit via a close encounter with 
Jupiter and if its pre-encounter perihelion distance was near Jupiter's orbit. 


\section{Conclusions}

We integrated the orbits of all the short-period comets (hereafter SPCs) in the Marsden (1989) Catalog under the influence of Sun and all the planets except Mercury and Pluto. The calculation was undertaken in order to study the dynamical lifetimes for these objects as as well as explain the current orbital element distribution. Four orbits were integrated for each comet, slightly varying their initial position. We found that the median dynamical lifetime of SPC is approximately 50000 years.

Our integration shows that Jupiter-family comets (JFCs. $P<20$ years) and Halley-family comets (HFC.s. $20<P<200$ years) are not dynamically distinct objects. Indeed, a comet can move between the families several times in its history. This is consistent with the results from several other authors (Lindren 1091, Nakamura \& Yoshikawa 1901) who performed integrations over much short periods of time. The perihelion and inclination of a comet (an also significantly change over its lifetime. It is not nncommon for a comet with a small perihelion to evolve onto an orbit with a q large enough so that it no longer is active. It is effectively stored in this orbit. Then after some time it can evolve back to small $q$ again. It can go through this process several times in its dynamical lifetime. On average a comet in the sample integrated here spends $53 \%$ of its time as a JFC and $64 \%$ of it time with $q<2.5 \mathrm{~A} U$.

The very flat inclination distribution of JFCs is observed to fatten up as it ages. This is clue to the inclination of individual comets increasing with time. If the present flat distribution is real and not an artifact of observational 
biases, then the natural explanation is that JFCs become extinct before their inclination distribution can change significantly. Our results predict that there should be a large population of extinct comets on high inclination JFC orbits. There is evidence for such objects. It has been shown that some of the meteors and meteor streams on JFCs orbits are found to have high inclinations (Olsson-Steel 1988). Indeed many are on retrograde orbits. This is also predicted by our integrations.

Most Jupiter-family comets are observed to have aphelion clistances very rlose to the semi-major axis of Jupiter. It is well known that most JFCs have recently suffered a close approach with Jupiter (Karm \& Rickman 10S2. Tincredi $S$ Lindgren 1992). They also tend to have argument of perihelions that are near 0 and $180^{\circ}$. We show that the later result is a direct consequence of the former if the orbit of the comet intersects the orbit of Jupiter. We also present an argument that the aphelion distance of a visible $(q<2.5 \mathrm{AU})$. JFC must be less than 6.4AU if it was captured onto its current orbit because of an encounter with Jupiter and if it previous orbit had a perihelion distance near the orbit of Jupiter.

We would like to thank E. Shoemaker. A. Stcrn, and P. Rimmeyer for useful discussions. We are particularly grateful to M. Duncan for collaborating on designing the code used for the N-body calculation. We would also like to thank S. Gauss and T. Corbin for supplying computer time to perform the N-body calculation. 


\section{References}

Belyaev, N. 1967, 'The Orbit Evolution of Comets Neujmin 2 (1916 II), Comas Solá (1027 III), Schwassmann-Wachmann 2 (1929 I) for 400 Years (1660-2060)', Astron.Zh., 44, 461.

Carusi A., Kresák, L., Perozzi, E., Valsecchi, G. 1985, Long-Term Evolution of Short-Period Comets (Adam Hilger: Bristol).

Carusi A., Valsecchi, G. 1987, 'Dynamical Evolution of Short-Period Comets' in Interplanetary Matter (eds. C'eplecha. Z. \& Pecina. P.). 21.

Duncan, M., Levison, H., \& Zingle, R. 1993, in preparation.

Duncan, M., Quinn. T., Tremaine, S. 1988. 'The Origin of Short-Period Comets', Astrophys. J. Letters, 328, L69.

Everhart, E. 1972, 'The Origin of Short-Period Comets'. Ap. Letters. 10. 131.

Fernández, J. 1980, 'On the Existence of a Comet Belt Beyond Neptune'. Mon.Not.Roy.Astron.Soc, 192, 481.

Fernández, J. 1985, 'Dynamical Capture and Physical Decay of Short-Period Comets', Icarus. 64, 308.

Fernández, J., Rickman. H., \& Kamél, L. 1992. 'The Population Size and Distribution of Perihelion Distances of the Jupiter Family' to appear in 
Proc. of the International Workshop on Periodic Comets (eds. Fernández. J. \& Rickman, H.).

Gladman, B.,\& Duncan, M. 1990, 'On the Fates of Minor Bodies in the Outer Solar System', Astron.J. 100, 1680.

Karm, J., \& Rickman, H. 1982, 'Pre-Discovery Encounters Between Short-Period Comets and Jupiter Estimated by Keplerian Approximation', Bull. Astron. Inst. Czechosl., 33, 359.

Kazimirchak-Polonskaya, E. 1967. 'Evolution des Orbites des Cométes á Courte Période au Cours des Années 1660-2060 et le Rôle des Planéts Extérieures Dans Cette Evolution', Astron.Zh., 44, 439.

Levison, H. 1991, 'The State of Kinowledge Concerning the Kuiper Belt' to appear in Asteroids, Meteriods, and Comets 1901. (eds. Harris, A. \& Bowell. E.)

Lingren, M. 1991, 'Dynamical Timescales in the Jupiter Family' to appear in Asteroids, Meteriods, and Comets 1991. (eds. Harris, A. \& Bowell. E.)

Lissauer, J. 1993, 'Planet Formation' to appear in Ann. Rev. Astron. Astrophy., 31.

Marsden, B. 1989, Catalog of Cometary Orbits (Hillside:Enslaw).

Nakamura, T., \& Yoshikawa, M. 1991, 'COSMO-DICE: Dynamical Investigation of Cometary Evolution', Publ. Natl. Astron. Obs. Japan 2, 293. 
Newton, H. 1893, 'On the Capture of Comets by Planets, Especially Their Capture by Jupiter: Mem. Natl. Acad. Sci. 6, T.

Olsson-Steel, D. 1988, 'Meteoroid Streams and the Zodiacal Dust Cloud' in Catastrophes and Evolution: Astronomical Foundations (ed Clube S.), 169.

Quinn, T., Tremaine, S., \& Duncan, M. 1990, 'Planetary Perturbations and the Origin of Short-Period Comets', Astrophys.J. 355, 667.

Rickman, H.. 1901. 'Plyysico-Dynamcial Evolution of Aging Comets' to appear' in the proceedings of the 1991 Goutelas Spring School of Astrophysics: Interrelations Entre la Physique et la Dynamique des Petits Corps dy Systéme Solaire

Shoemaker, E. 1992, Private communication.

Shoemaker, E., \& Wolfe. R. 19S2, 'Cratering Time Scales for the Galilean Satellites' in Satellites of Jupiter (ecl. Marrison, D.), 277.

Stagg, C., \& Bailey, M. 1989, 'Stochastic Capture of Short-Period Comets', Mon.Not.Roy.Astron.Soc. 241, 50T.

Tancredi, G., \& Rickman, H. 1992, 'The Evolution of Jupiter Family Comets over 2000 Years', in Chaos, Resonance and Collective Dynamical Phenomena in the Solar System (ed. Forraz-Mello, S.), 269.

Tancredi, G., Lingren, M. 1991, 'The Vicinity of Jupiter: A Region to Look for 25 
Comets' to appear in Asteroids, Meteriods, and Comets 1991. (eds. Harris.

A. \& Bowell, E.)

Wisdom. J.,\& Holman, M. 1901, 'Symplectic Maps for the N-Body Problem', Astron.J. 102, 1528. 


\section{Captions}

Figure 1 - The fraction of short-period comets with lifetimes greater then a particular time as a function of time.

Figure 2 - The relationship between inclination, $i$, and semi-major axes, $a$, for all short-period comets in the Marsden (1989) Catalog. The opened and filled circles represent comets with $q \leq 1 A U$ and $q>1 . A U$, respectively. The dotted line represents the division between JFCs and HFCs.

Figure 3 - The orbital element distribution of Jupiter-family comets. a) Histogram of the aphelion distance. The dotted line shows $5.2 . A U$. b) Histogram of the argument of perihelion.

Figure 4 - The behavior of comet P/Parker-Hartley over its entire dynamical history. a) Semi-major axis. The rotted line represents the division between JFCs and HFC's. b) Perihelion distance. The dotted line represents the limit of visibility set by Duncan. Quinn. S Tremaine (1988). c) Inclination. The dotted line represents $i=40^{\circ}$. a) Argument of perihelion.

Figure 5 - The mean cosine of the inclination of comets remaining as a function of time for both the forward and backward integrations. a) All comets. b) Jupiter-family comets. The solid curve is all JFCs. The dotted curve is just those JFCs with $q \leq 2.5 \mathrm{~A} U$. 
Figure 6 - a) The mean cosine of the inclination of all comets within a perihelion bin as a function of the bin's mean $q$. The filled circles represent the mean for all comets within the bin. while the open circles represent the mean for only those comets the are on direct orbits. b) The mean cosine of the inclination of a individual comet as a function of its dynamical lifetime. c) The time averaged $\cos (i)$ of an individual comet as a function of its current observed $\cos (i)$.

Figure $\vec{\imath}$ - The orbital element distribution of Jupiter-family comets at three times in our integration. The solid histogram shows the current distribution. The dotted histogram represents the distribution averaged over 2000 years centered on 5000 years in the future and dashed histogram is averaged over 20000 years centered on 50000 ycars in the future. The arrows represent the expected range from the theory. a) Histogram of the aphelion distance. b) Histogram of the argument of perihelion.

Figure 8 - Results from our simple capture scenario. a) The final aphelion distance of a comet captured into a JFC orbit as a function of the final perihelion distance for three different initial semi-major axes. 1) Sane ats a except the final $\left|\omega+\omega_{\text {off }}\right|$. 


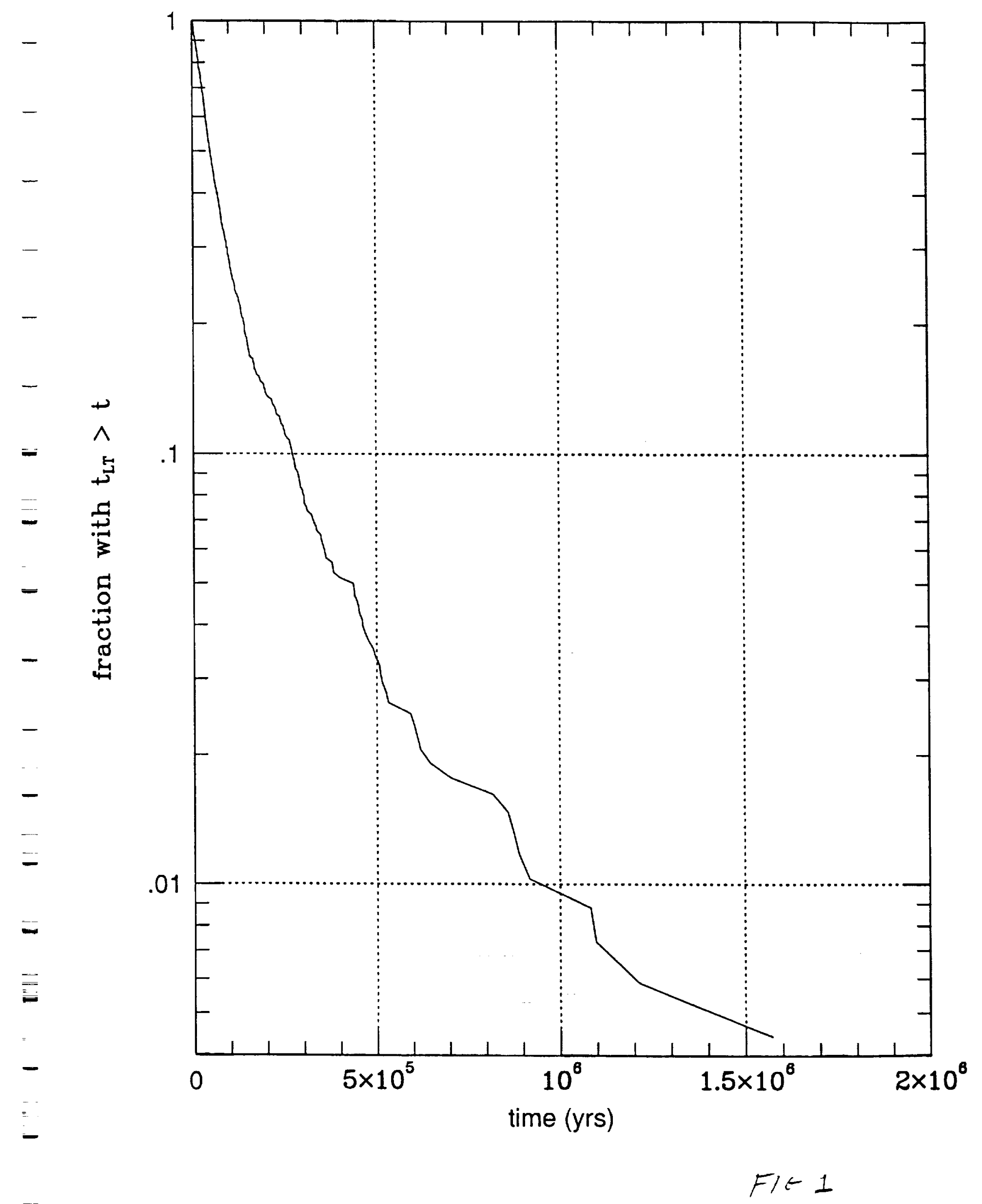




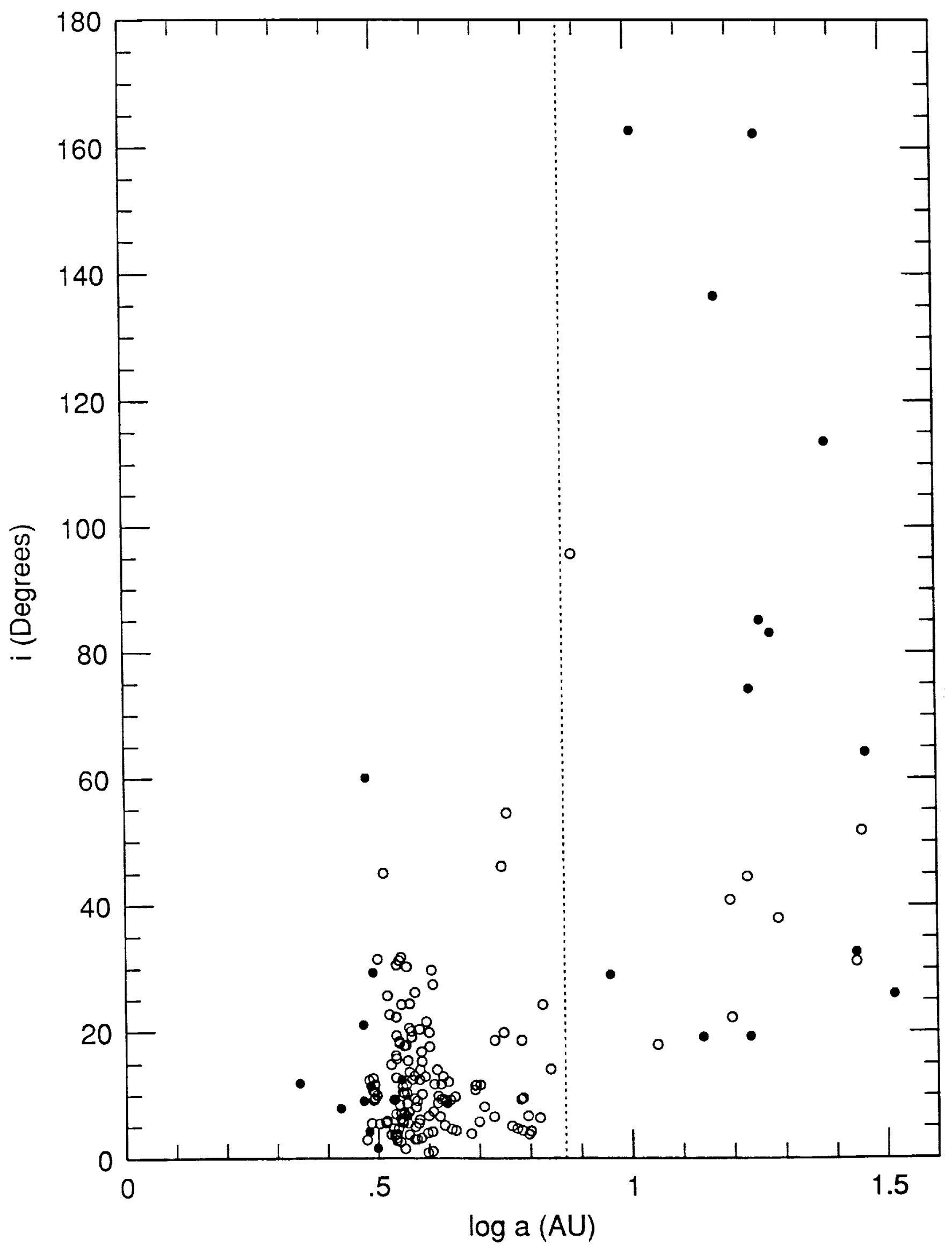

FIG 2 

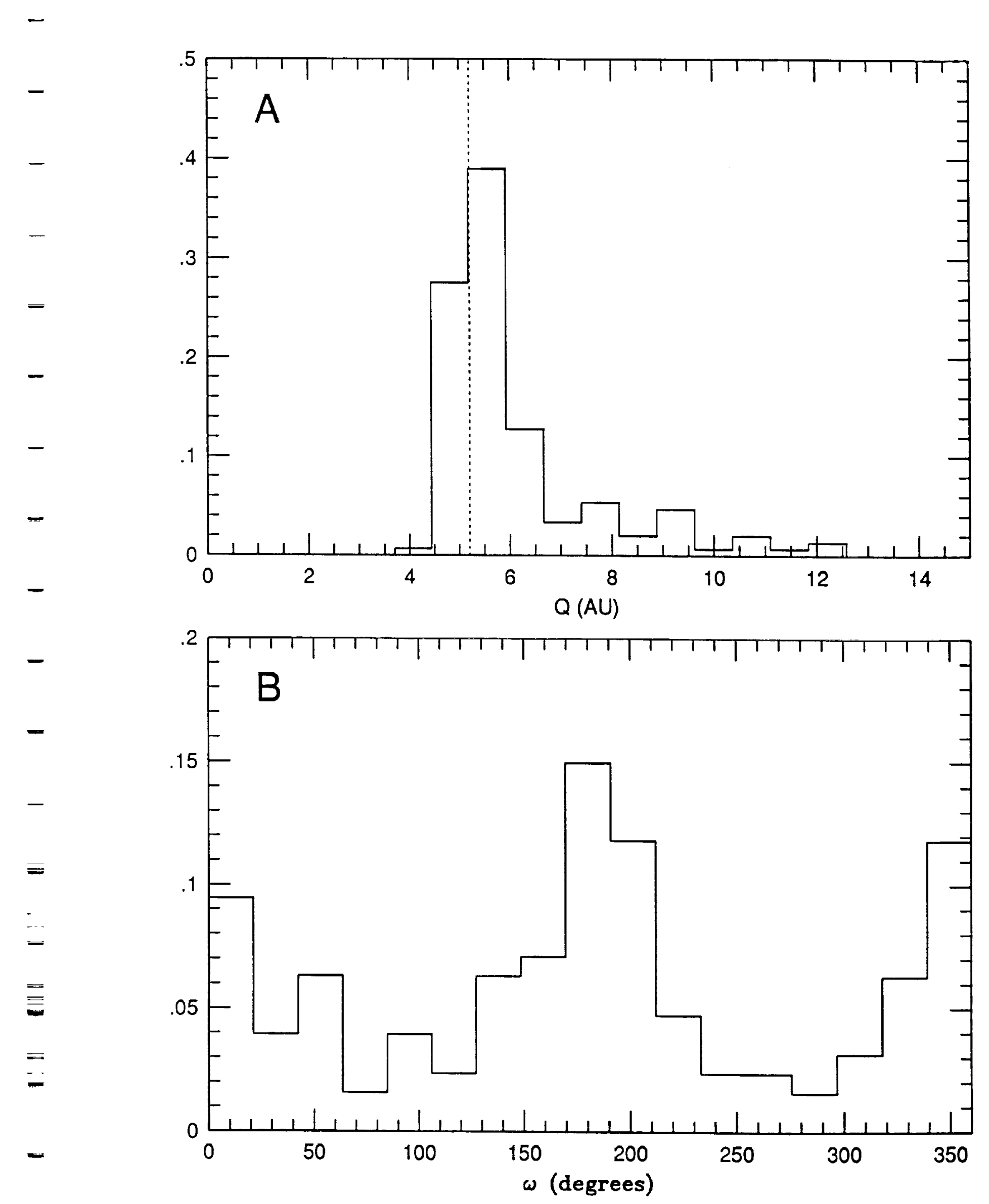

FIE 3 

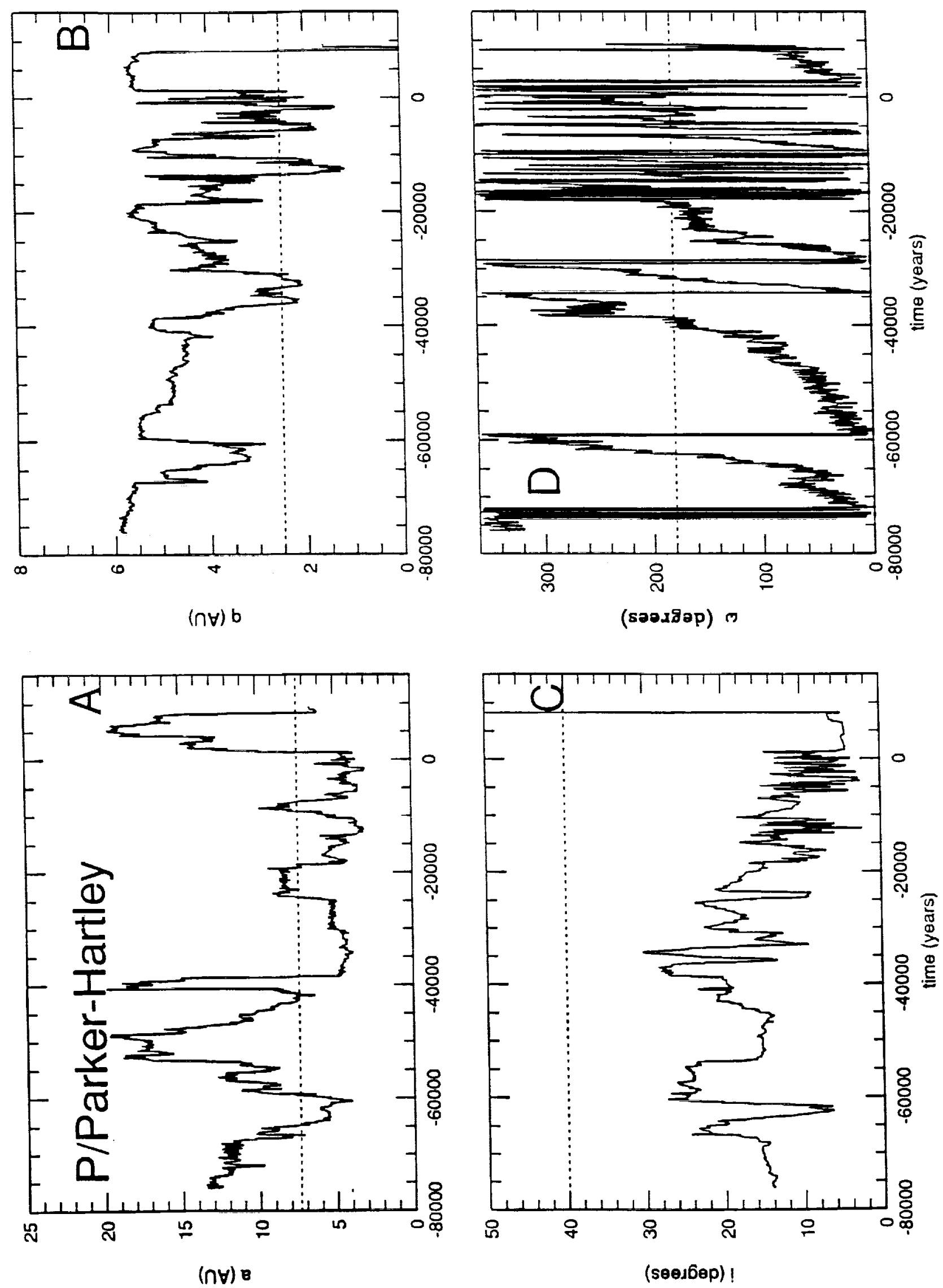

FIG 

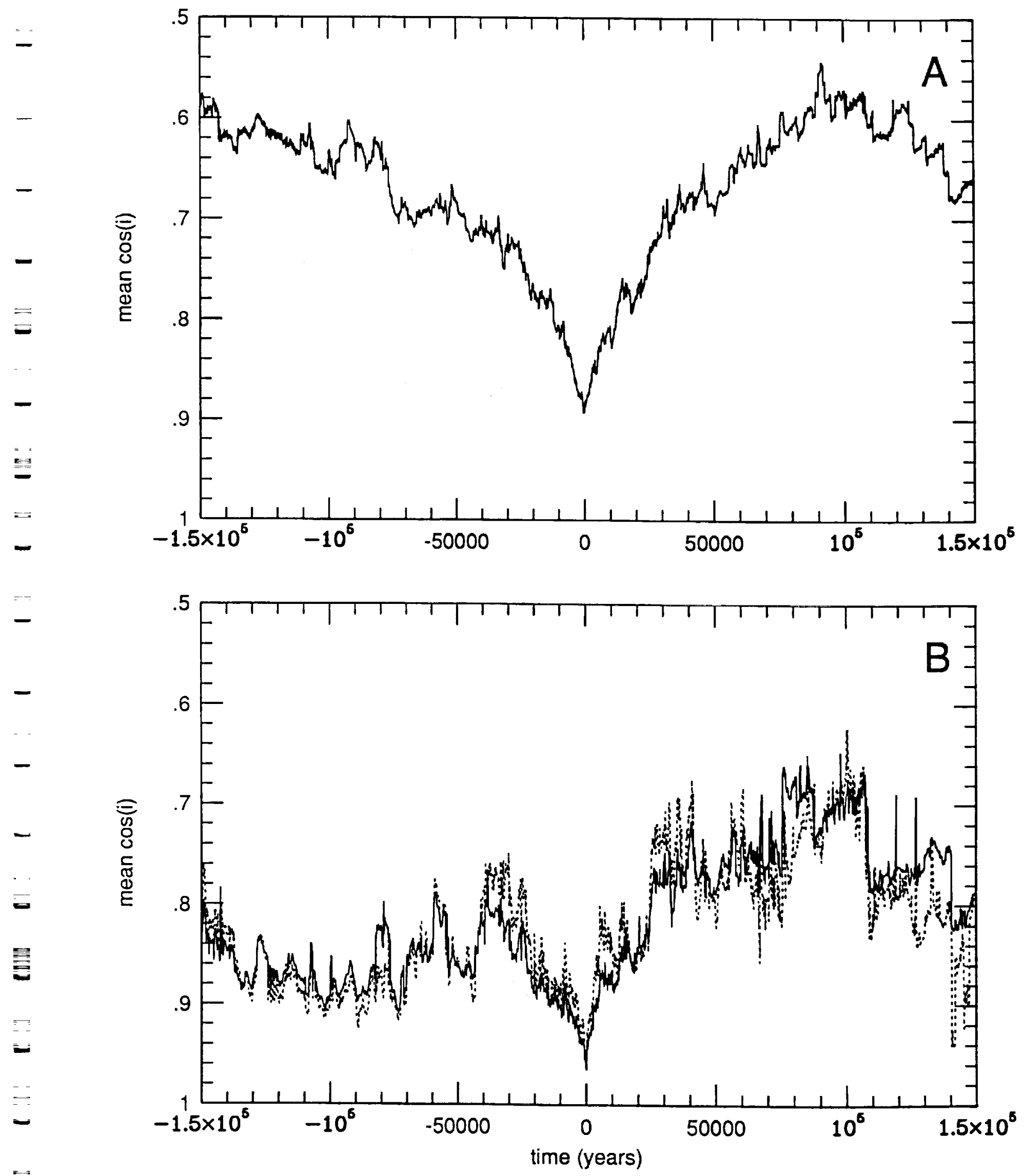

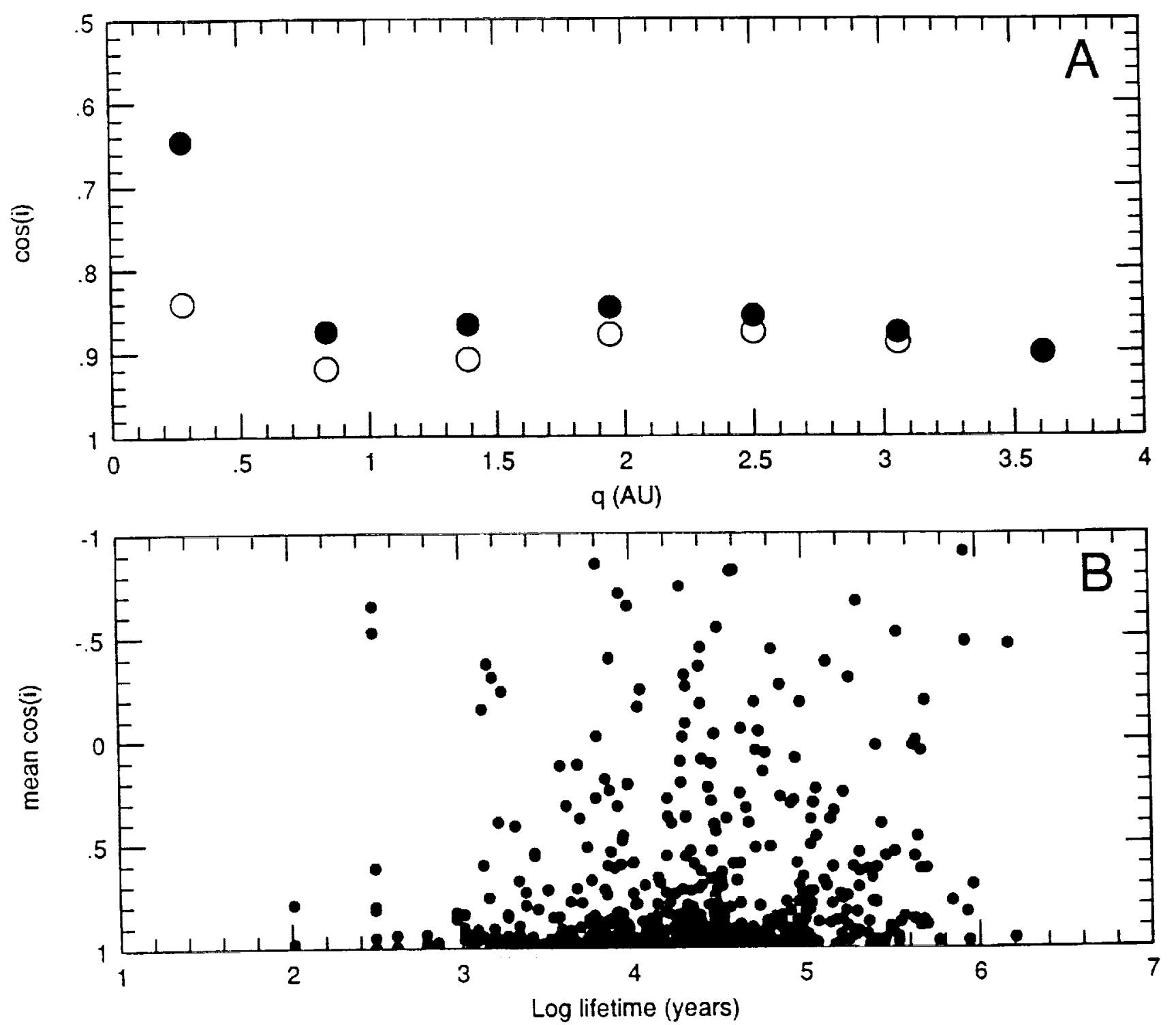

$\bar{E}$

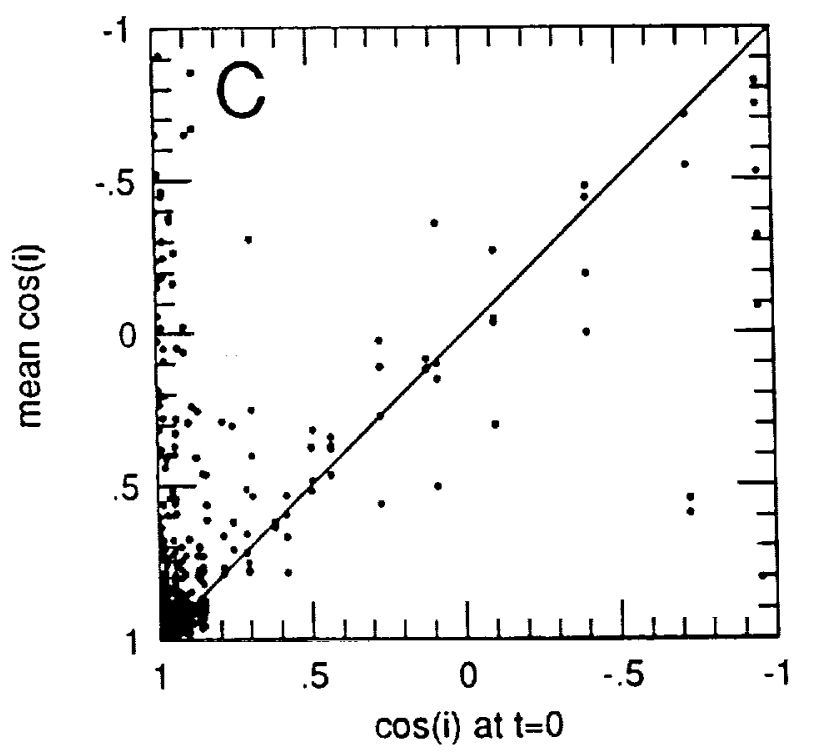

FIE 6 

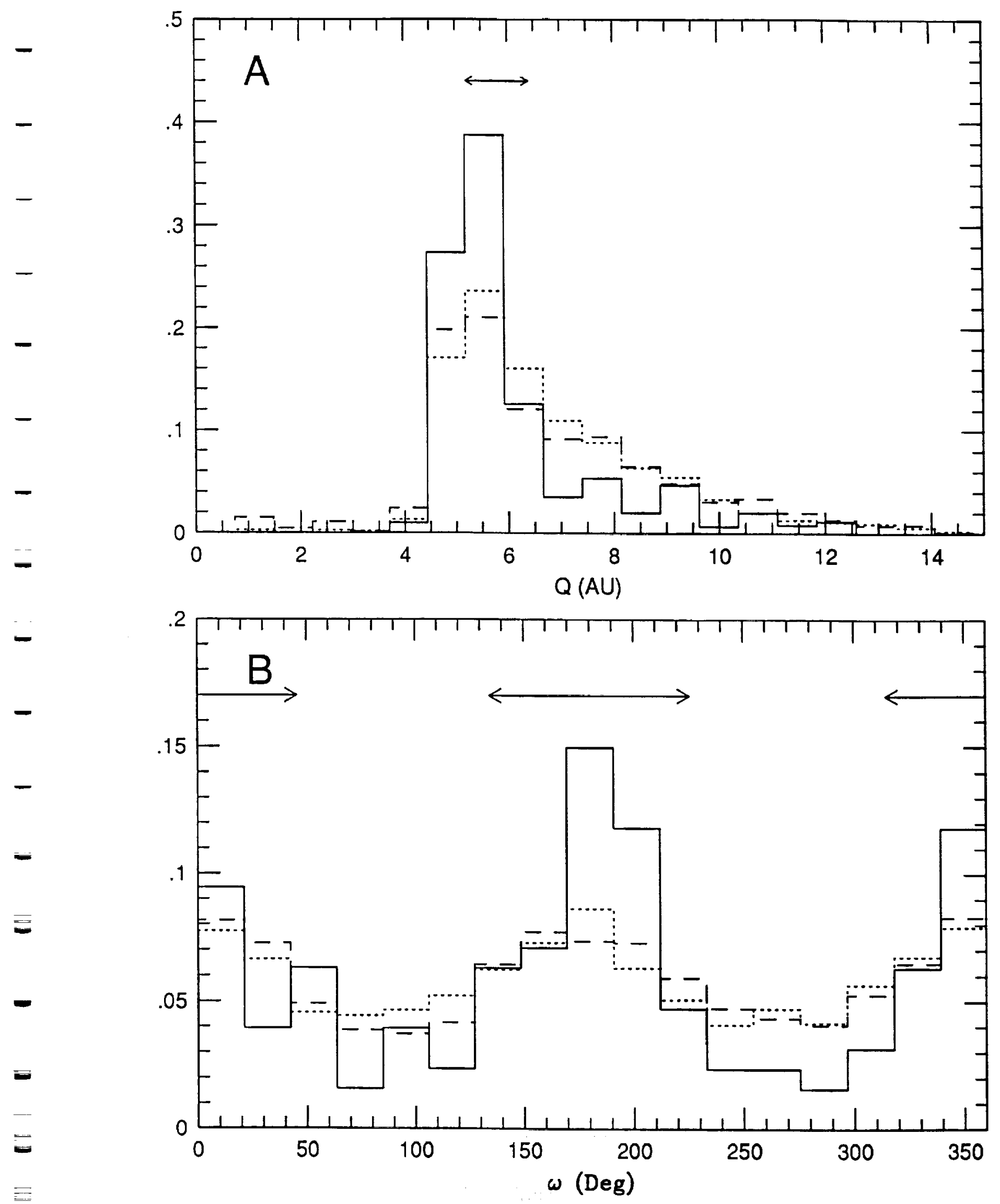

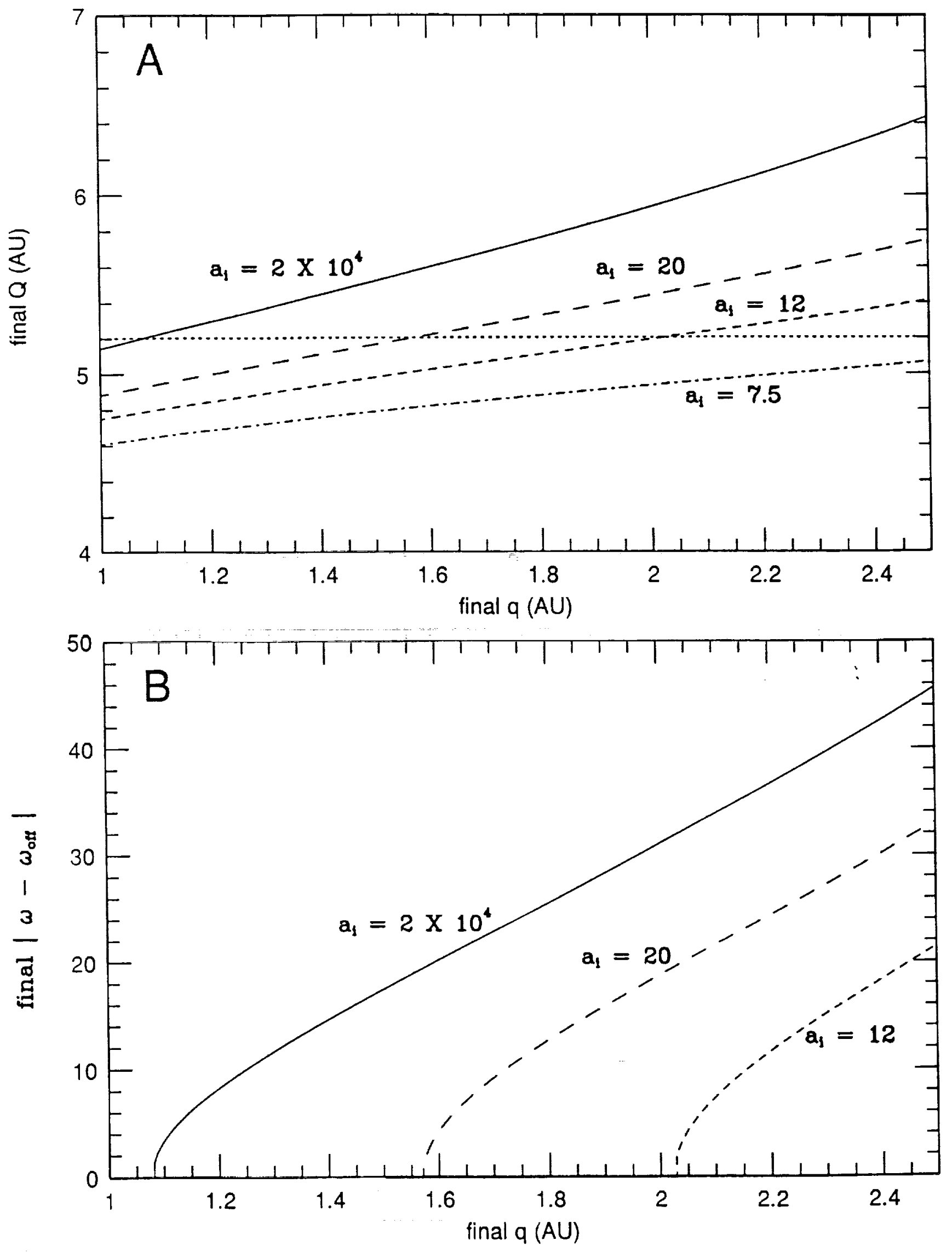

$\therefore \leq-?$ 